\title{
The dorsal premotor cortex exerts a powerful and specific inhibitory effect on the ipsilateral corticofacial system: a dual-coil transcranial magnetic stimulation study
}

\author{
Sara Parmigiani $^{1} \cdot$ Guido Barchiesi $^{1} \cdot$ Luigi Cattaneo $^{1}$
}

Received: 20 April 2015 / Accepted: 20 July 2015

(C) Springer-Verlag Berlin Heidelberg 2015

\begin{abstract}
A rich pattern of connectivity is present in non-human primates between the dorsal premotor cortex (PMCd) and the motor cortex (M1). By analogy, similar connections are hypothesized in humans between the PMCd and the ipsilateral hand-related M1. However, the technical difficulty of applying transcranial magnetic stimulation (TMS) with a dual-coil paradigm to two cortical regions in such close spatial proximity renders their in vivo demonstration difficult. The present work aims at assessing in humans the existence of short-latency influences of the left PMCd on the ipsilateral corticofacial system by means of TMS. A dual-coil TMS paradigm was used with 16 participants. Test TMS pulses were applied to the left orofacial M1, and conditioning TMS pulses were applied to three distinct points of the ipsilateral PMCd along the caudal part of the superior frontal sulcus. The inter-stimulus interval (ISI) between condTMS and testTMS varied in 2-ms steps between 2 and $8 \mathrm{~ms}$. Motor evoked potentials (MEPs) in the active orbicularis oris muscle were recorded. CondTMS exerted a robust effect on the corticofacial system only when applied to one specific portion of the PMCd and only at one specific ISI (6 ms). The effect consisted in a systematic suppression of facial MEPs compared to those obtained by testTMS alone. No other effect was found. We provide evidence for a specific short-latency inhibitory effect of the
\end{abstract}

Electronic supplementary material The online version of this article (doi:10.1007/s00221-015-4393-7) contains supplementary material, which is available to authorized users.

Luigi Cattaneo

luigi.cattaneo@unitn.it

1 Centro Interdipartimentale Mente/Cervello (CIMeC), Università di Trento, Via delle Regole 101, 38123 Trento, Italy
PMCd on the ipsilateral M1, likely witnessing direct corticocortical connectivity in humans. We also describe a novel paradigm to test ipsilateral PMCd-M1 in humans.

Keywords Motor cortex · Facial movements - Premotor cortex $\cdot$ Decisions $\cdot$ Connectivity $\cdot$ Action selection

\section{Introduction}

The portion of cortex lying rostral to the motor cortex is commonly referred to as premotor cortex (PMC) (Fulton 1935; Vogt and Vogt 1919). Its microscopic features are intermediate between the agranular pattern found in the motor cortex and the granular pattern found in the prefrontal cortex. The PMC of primates is part of the cortical motor system (Rizzolatti et al. 1998). It can therefore generate motor outputs directly by means of corticospinal or corticobulbar projections (Dum and Strick 1991; He et al. 1993, 1995; Morecraft et al. 2001) or indirectly by means of a rich pattern of connections to the motor cortex (M1). Odological data in non-human primates indicate massive anterograde projections from the PMC to the hand-related motor area (handM1) in the macaque (Dum and Strick 2005; Hatanaka et al. 2001; Muakkassa and Strick 1979; Tokuno and Tanji 1993). More generally, the PMC-M1 module is involved in the generation and control of upper limb movements (Kraskov et al. 2011). Indirect anatomical data suggest that in non-human primates, hind limb and mouth movements may also be controlled by PMC-M1 circuits (Hatanaka et al. 2001; Morecraft et al. 2001).

In humans, the PMC coincides with Brodmann's area 6 (Brodmann 1909). This area has been divided into three main sectors: the medial premotor cortex, the dorsal premotor cortex (PMCd) and the ventral premotor cortex (PMCV). 
The connectivity between the PMC and the ipsilateral M1 in humans is largely hypothesized by analogy with the non-human primates. Still, direct evidence for it has been obtained by means of the dual-coil paradigm of transcranial magnetic stimulation (TMS) (Baumer et al. 2009; Cattaneo and Barchiesi 2011; Civardi et al. 2001; Davare et al. 2008, 2009, 2010; Koch et al. 2006; Mars et al. 2009). This protocol involves the use of two magnetic stimulators connected to two distinct coils, both of which are used simultaneously on the participant's scalp. One coil (testTMS) is placed over the M1 and delivers supra-threshold stimuli, ultimately generating motor evoked potentials (MEPs). Prior to the testTMS stimulus, a conditioning stimulus (condTMS) is delivered by a second coil. The second coil is placed over a cortical area that is hypothetically connected to the M1. If the prior delivery of condTMS were to change the amplitude of MEPs generated by testTMS, this would indicate that there is an influence of the cortical area on the M1 (Rothwell 2011).

The main technical difficulty involved in applying the dual-coil paradigm arises from the close proximity between the target areas of condTMS and a testTMS over the handM1 (Johansen-Berg et al. 2002; Schluter et al. 1998). In the case of the PMCv and of the medial premotor cortex, this distance still allows the use of small commercially available coils. In the case of the PMCd, the actual distance from the handM1 cortex is too short to allow either coil placement or selective stimulation of either cortical region. Indeed, functional imaging studies indicate that the focus of activity during conditional sensorimotor behavior (a task that is supposedly carried out by $\mathrm{PMCd}$ ) is around $1.5-2 \mathrm{~cm}$ from the ipsilateral handM1 (Amiez et al. 2006; Fink et al. 1997; Schluter et al. 1998). Most researchers have resorted to testing the contralateral PMCd with testTMS to solve this problem (Baumer et al. 2009; Koch et al. 2006; Mochizuki et al. 2004; O'Shea et al. 2007a, b). Alternatively, the use of special non-commercial coils has been attempted (Groppa et al. 2012); this solves the problem of simultaneous coil placement but does not fully allow selective stimulation of either target.

In the present work, we explore the possibility of using a dual-coil paradigm to test ipsilateral PMCd-M1 connectivity by changing the target of testTMS. Instead of the handM1, we stimulate the mouth-related M1 (mouthM1), which is located ventrally. This approach solves both the problem of simultaneous coil placement and that of selective stimulation of the two target areas.

\section{Methods}

\section{Participants and general protocol}

Sixteen healthy volunteers (11 women, mean age 25.13, range 19-32, SD 3.76) took part in this study. All gave written informed consent to the experiment, and none had contraindications to TMS (Rossi et al. 2009). This study was approved by the University of Trento Ethical Committee (protocol 2031-032) and conducted in compliance with the revised Helsinki declaration (World Medical Association General Assembly 2008).

The present work aimed at assessing the short-latency influences of the left PMCd on the ipsilateral corticofacial system with the dual-coil technique. CondTMS was applied to the PMCd, and testTMS was applied to the orofacial motor cortex (mouthM1). The scalp projection of the mouthM1 was functionally localized as the spot where highest amplitude MEPs were elicited with the minimal intensity. On the contrary, there was no clear a priori hypothesis on the PMCd topography, and therefore, three different points were tested in the standard positions of the Brodmann area 6 along the superior frontal sulcus (sFS). The three points were identified on individual anatomical MRI scans by means of frameless stereotaxic neuronavigation. Participants were tested during active contraction of the lips but with no other active task. MEPs in the facial region may be difficult to obtain at rest because of high threshold of the orofacial motor cortex to TMS (Cattaneo and Pavesi 2013). It is therefore common practice to record facial MEPs during active contraction of the target muscle.

\section{Neuronavigation}

For each subject, a structural MRI scan was acquired before the experiment to allow MRI-neuronavigated positioning of the condTMS. A high-resolution T1-weighted magnetization prepared rapid gradient echo sequence (176 axial slices, in-plane resolution $256 \times 224,1-\mathrm{mm}$ isotropic voxels, generalized autocalibrating partially parallel acquisition with acceleration factor $=2$, time repetition $=2700 \mathrm{~ms}$, time echo $=4.180 \mathrm{~ms}$, time to inversion $=1020 \mathrm{~ms}$, flip angle $=7 \mathrm{u}$ ) scan of the brain of each subject was obtained using a MedSpec 4-T head scanner (Bruker BioSpin $\mathrm{GmbH}$, Rheinstetten, Germany) with an eight-channel array head coil. Starting from this scan, a 3D reconstruction of the scalp and the gray matter surfaces was produced using MesH morphing tool included in the BrainVoyager software (Brain Innovation BV, The Netherlands). The BrainVoyager neuronavigation software combined with an ultrasound tracking system, CMS205S (Zebris Medical GmbH, Isny, Germany) was used to coregister the 3D scalp reconstruction with the actual participant's head, thus marking the target points for TMS on the real head.

\section{Identification of condTMS and testTMS target areas}

In each participant, three different stimulation points over the putative dorsal premotor region were identified. The 
three spots will be referred to as P1-P3 and were identified on the basis of macro-anatomical landmarks. P1 was located corresponding to the junction between the superior precentral sulcus (sPreCS) and the sFS. The two other spots were located along the sFS. P2 was located $1.5 \mathrm{~cm}$ rostral to $\mathrm{P} 1$, and $\mathrm{P} 3$ was located $3 \mathrm{~cm}$ rostral to $\mathrm{P} 1$. Variability in the morphology of the precentral sulcal pattern of this region is subject to considerable inter-individual variations (Germann et al. 2005) and therefore needed accurate participant-by-participant investigation. In certain participants, the sPreCS consisted in one continuous sulcus, whereas in others, it is composed of two separate folds. In each participant, the pattern was correctly identified and $3 / 16$ had a discontinuous sulcus. The sFS was separated from the sPreCS in $8 / 16$ of cases. The minor sulci, namely the medial precentral $(\mathrm{MeP})$ and the caudal paramidline (PaM) sulci, were identified so that no confusion with the sFS proper could be made. All the participants' anatomy is shown in Fig. 1. The position of the three spots was planned to cover in a caudo-cranial direction the whole span of dorsal BA6 (Brodmann 1909; Geyer 2004)—see "Discussion". TestTMS was applied to the motor cortex at the point where largest MEPs could be elicited from the orbicularis oris (OOr) muscle.
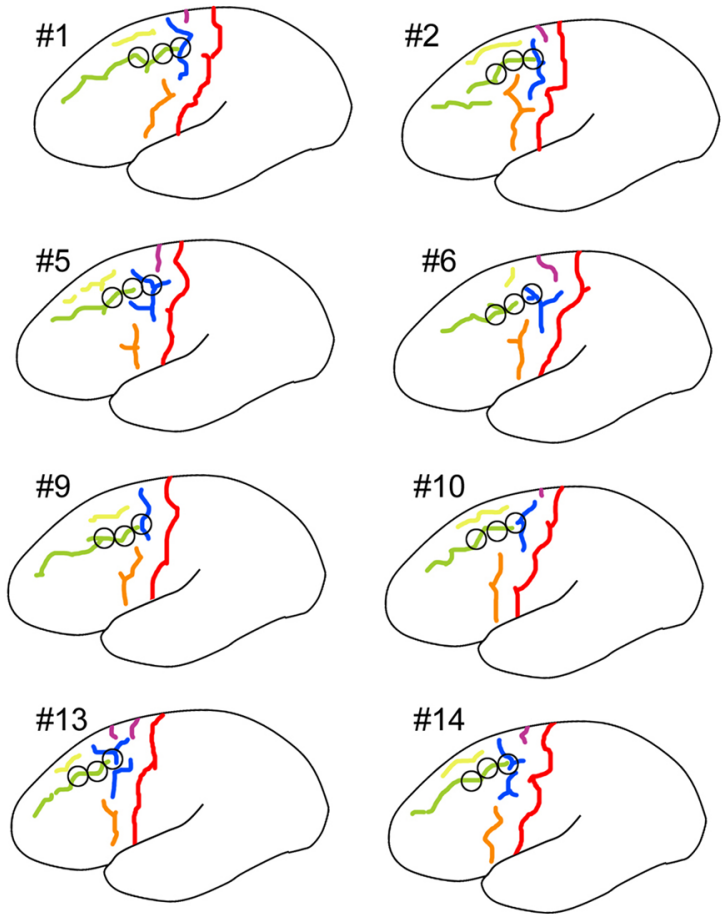

Fig. 1 Schematization of the anatomy of the precentral sulcal complex in all 16 subjects. Red central sulcus; blue superior precentral sulcus; orange inferior precentral sulcus; green superior frontal sul-

\section{EMG recordings}

The right side of the OOr and the right first dorsal interosseus (1DI) were recorded with surface electrodes. On the OOr, the two electrodes were placed parallel to the muscle fibers on the lower lip (see Supplementary Figure 1). The analog signal was amplified $1000 \times$ and band-pass-filtered between $5 \mathrm{~Hz}$ and $2 \mathrm{kHz}$ and by means of a 1902 two-channel amplifier (Cambridge Electronic Design, Cambridge, UK).

Participants were given a stick to be held in their mouth with their lips only and were asked to generate muscular tension matching an average amplitude of the EMG signal of around $200 \mu \mathrm{V}$. The operator assisted them in finding the desired amount of contraction and monitored it during the whole experimental session. Furthermore, they could see the EMG signal on the screen of the computer and had to remain with their contraction into the boundaries of the two cursors indicating the required level of contraction (e.g., -0.1 and $0.1 \mathrm{mV}$ ). The analog EMG signal was then digitalized (with a sampling frequency of $4 \mathrm{kHz}$ ) by means of a 1401 micro Mk-II unit (Cambridge Electronic Design). Recordings and triggers were dealt with via the Signal software (Cambridge Electronic Design). The digitized EMG
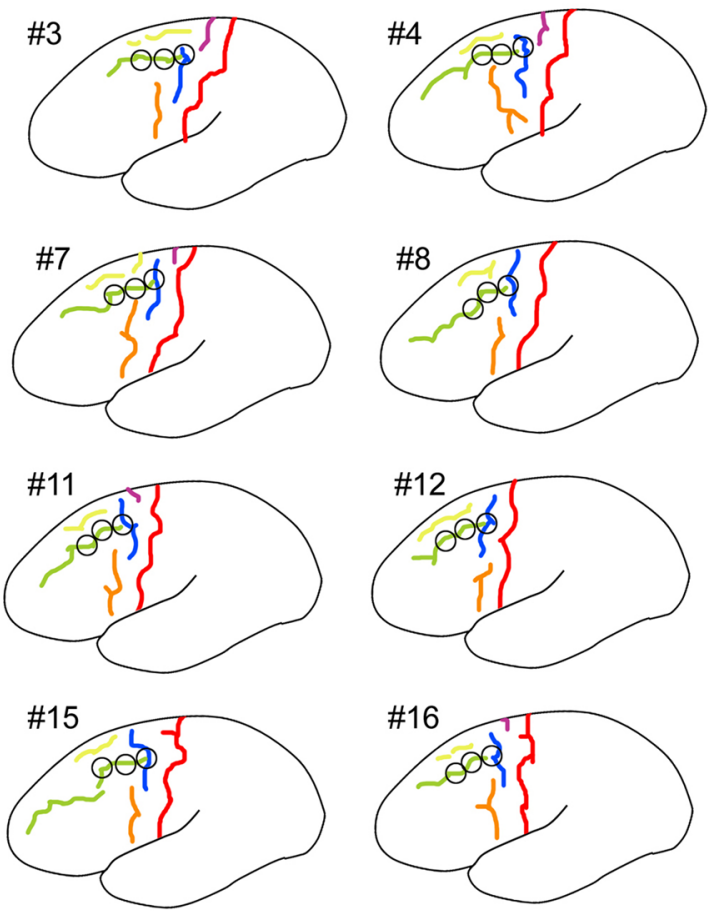

cus; purple medial precentral sulcus; yellow paramidline sulcus. The individual localization of the three stimulation points, P1-3, is indicated with hollow circles (color figure online) 
was acquired in epochs of $500 \mathrm{~ms}$, i.e., from $200 \mathrm{~ms}$ before to $300 \mathrm{~ms}$ after the testTMS pulse.

\section{TMS}

Participants wore earplugs and were sitting comfortably with their head on a chin rest and with an additional lateral head constraint, which was adjusted individually to allow for a comfortable posture as well as to assure head stability and minimal movement. They kept their eyes freely open and had to stay physically inactive except for the contraction of the muscles that enabled them to hold a stick between their lips firmly. Two magnetic stimulators were used in this experiment. The one delivering the testTMS to M1 was a MagPro stimulator (Medtronic, Denmark), connected to a figure-of-eight coil with 55-mm windings (Dantec B55, Skovlunde, Denmark), oriented perpendicularly to the midline with the handle pointing medially. The one delivering the condTMS over the PMCd was a MagPro Compact (MagVenture, Skovlunde, Denmark), connected to an MC-B35 figure-of-eight coil with windings of $35 \mathrm{~mm}$ diameter (MagVenture, Skovlunde, Denmark); the condTMS coil was positioned tangentially to the scalp in varied orientations according to the mechanical interaction between the two coils. Both the testTMS and the condTMS coils were held by an articulated mechanical arm (Manfrotto 244, VitecGroup, Italy). The position of the condTMS coil was determined by frameless stereotaxic neuronavigation (see above).

The intensity of the condTMS as well as the testTMS stimuli was around $120 \%$ of the active motor threshold (AMT) of the OOr and set on a subject-by-subject basis. AMT was defined as the minimum stimulus intensity required to produce a MEP in the recorded muscle of approximately $200 \mu \mathrm{V}$ in five out of 10 consecutive trials during a mild voluntary contraction.

\section{Inter-stimulus intervals (ISIs) between condTMS and testTMS}

In each trial, TMS could be delivered either as a single testTMS pulse (single-pulse trials) or as the combination of condTMS + testTMS (dual-pulse trials). Five different ISIs were used: 8, 6, 4, 2 and $-1 \mathrm{~ms}$ (the negative sign indicates that condTMS was delivered after testTMS in this single ISI). Single-pulse trials were interleaved with dualpulse trials, as shown in Fig. 2. Dual-pulse trials of a given ISI were repeated 12 times for each of the three points of stimulation. Ultimately, a total of 96 trials was associated with each stimulation point: 36 single-pulse trials and 60 dual-pulse trials.

\section{MEP data preprocessing}

The EMG signal was preprocessed according to the following steps: (1) the signal was high-pass-filtered at $20 \mathrm{~Hz}$. (2) The EMG was rectified. (3) The area under the curve in the time window between 10 and $30 \mathrm{~ms}$ after the testTMS stimulus was extracted. The particular time window was chosen to cover the duration of MEPs in the orofacial region (Cattaneo and Pavesi 2013). (4) The baseline EMG activity was defined as the area under the rectified EMG signal in the $200 \mathrm{~ms}$ prior to condTMS and was extracted for each trial. (5) Given that MEP amplitudes covariate strictly with the background EMG activity, we performed a baseline correction by dividing the MEPs areas by the baseline in individual trials. The procedure of baseline correction is already known in the literature to deal with the variability of MEP amplitudes from cranial muscles during active contraction (Sato et al. 2010; Watkins and Paus 2004; Watkins et al. 2003).

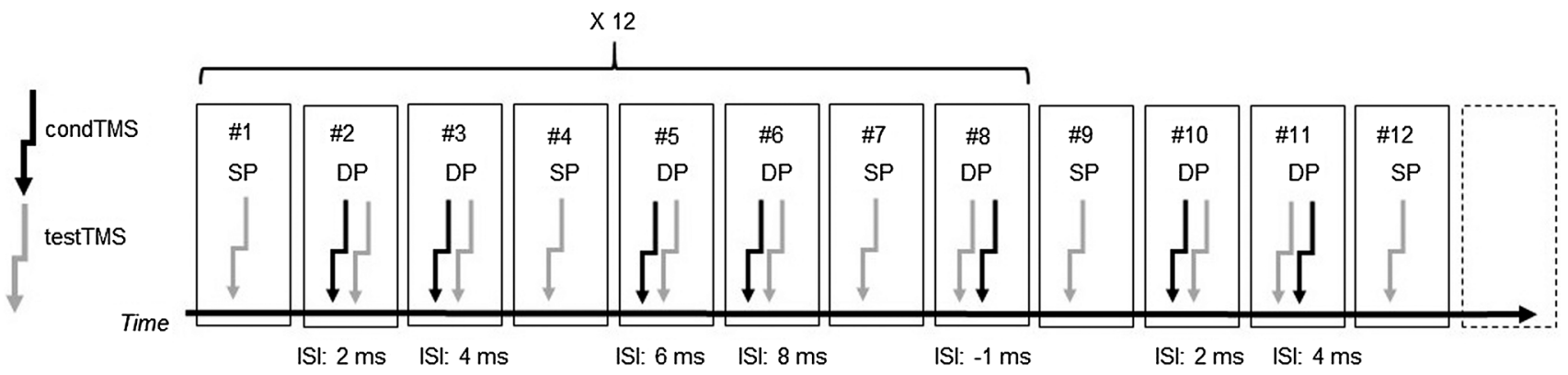

Fig. $2 S P$ single-pulse, $D P$ dual-pulse, $I S I$ inter-stimulus interval; a schematic representation of the SP trials interleaved with DP trials. Each ISI were repeated 12 times for each of the three points of stimulation, for a total of 96 trials with each stimulation point (36 single- pulse trials and 60 dual-pulse trials). Normalization procedure: trials \#2 and \#3 were divided by the average of trials \#1 and \#4, trials \#5 and $\# 6$ by the average of trials \#4 and \#7, trial \#8 by the average of trials \#7 and \#9 and so on 


\section{Normalization of dual-pulse MEPs}

Dual-pulse MEPs were normalized to single-pulse MEPs. To do so, a procedure of normalizing single trials of dualpulse MEPs to the average of single-pulse MEPs in a sliding window that followed the dual-pulse trials was adopted (Cattaneo and Barchiesi 2011; Maule et al. 2015). The two single-pulse trials immediately adjacent to the dual-pulse trial were used as the sliding window. Their value was averaged and was used as a denominator in a ratio in which the numerator was the value of the dual-pulse MEP in between the two single pulses, thus obtaining a normalized index. The normalization procedure is schematized in Fig. 2. The resulting data are a ratio and therefore are distributed between 0 and $+\infty$. This distribution is by definition not normal. In order to achieve normality of the data, we applied a further manipulation, i.e., a base ten logarithmic transformation, to each value (Tukey 1977). In this way, data were symmetrically distributed around 0 , between $-\infty$ and $+\infty$. Individual pools of data were then successfully tested for normality by means of Shapiro-Wilk's test. In this novel distribution of data, negative values indicated amplitude of dual-pulse MEPs smaller than the instantaneous value of MEPs from single-pulse alone, whereas positive values indicated amplitude of dual-pulse MEPs larger than the instantaneous value of MEPs from single-pulse alone. In conclusion, the final result of the procedure was a series of 60 normalized dual-pulse MEPs for each stimulation point.

\section{Statistical analysis of normalized MEPs}

The normalized MEPs were used as dependent variable in an ANOVA for repeated measures with two within-subjects factors. The TARGET factor indicated which point had been stimulated with condTMS and had three levels: P1, P2 or P3. The ISI factor had five levels corresponding to each of the five different ISI between condTMS and testTMS. Post hoc analyses were conducted with Newmann-Keuls' test.

\section{Control analyses}

All MEP amplitudes were corrected by the baseline EMG activity prior to TMS, as described above. However, to rule out the possibility that baseline EMG could be non-randomly distributed between the different experimental conditions, thus producing a bias, we analyzed the pre-stimulus EMG area of the $200 \mathrm{~ms}$ prior to TMS in a TARGET * ISI ANOVA.

The analysis of the dual-pulse condition by normalizing with the sliding window is relatively novel (Cattaneo and Barchiesi 2011). Hence, we decided post hoc to perform, alongside to the main analysis, a conventional analysis based on averaging all MEPs within conditions and computing the ratio between the grand averages of the dualpulse trials and that of the single-pulse trials as is generally done in dual-coil TMS experiments (Davare et al. 2009; Koch et al. 2006; Mars et al. 2009; O'Shea et al. 2007b). For each subject, we thus obtained 15 values (three TARGETS $\times$ five ISIs) values of the single-pulse/dual-pulse MEPs ratio. This ratio was higher than 1 if facilitation had occurred or lower than 1 if inhibition had occurred. We therefore used $t$ tests for single samples to test the hypothesis that the mean values of the ratio were different from 1 .

\section{Results}

None of the subjects reported undesired effects of TMS. In all participants, a repeatable and consistent MEP was obtained from the activated OOr muscle. The mean active motor threshold for the OOr muscle was $60.3 \%$ $(\mathrm{SD}=3.8 \%)$ of the stimulator's output. The mean stimulation intensity was therefore $72.5 \%(\mathrm{SD}=4.5 \%)$, ranging between a minimum of $65 \%$ to a maximum of $80 \%$. Figure 3 displays a representative recording from the OOr of one subject. A MEP in the 1DI was observed in six subjects, and limitedly to dual-pulse trials with the condTMS coil over P1. Since the 1DI MEPs were limited to the dual-pulse trials (no MEPs in the 1DI were obtained in the single-pulse trials, even in the P1 trials), it is highly plausible that they are due to the condTMS over the P1, considered the close proximity of this portion of area

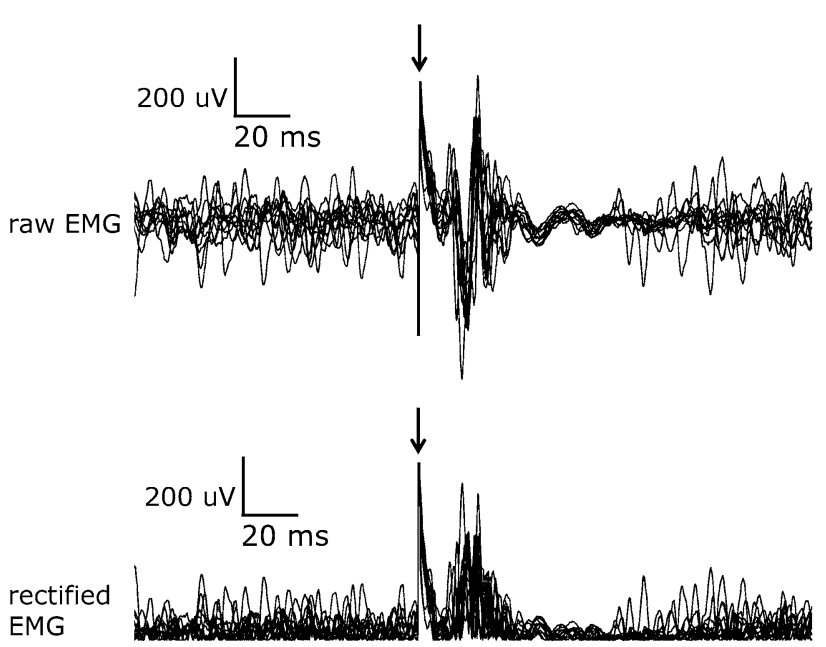

Fig. 3 Representative EMG recordings from the orbicularis oris muscle of one subject. Ten consecutive recordings from single-pulse trials are shown. The upper panel the raw EMG data and the lower panel the data after rectification of the signal, prior to extraction of the MEP area. The arrows the time of TMS over the mouth motor cortex 


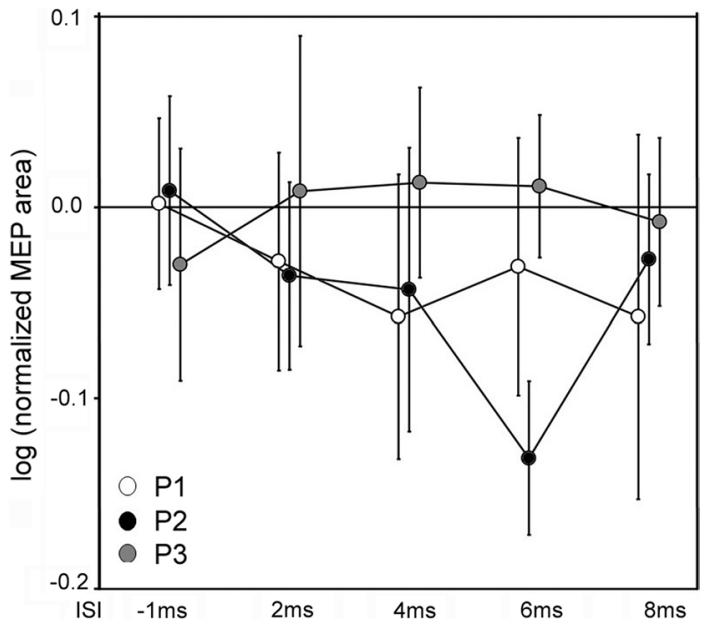

Fig. 4 Representation of the experimental results. The average values $(n=16)$ of the logarithm of the normalized MEP areas subjects are shown for each of the ISI and each of the target points. Error bars indicate $95 \%$ confidence intervals

6 with the handM1. This is in line with the drawback of dual-pulse technique applied over the PMCd and the ipsilateral handM1, which not always allows a selective stimulation, and corroborate our initial purpose to stimulate the mouthM1. It could be speculated that $72 \%$ of stimulator output is too high an intensity to guarantee focality of stimulation. Indeed other authors have found corticocortical connectivity to M1 from the supplementary motor area (Arai et al. 2012), which is not far from the dorsal premotor region over which we applied the condTMS. However, the coils that we used for condTMS were considerably smaller (35 mm of outer diameter) than conventional coils, thereby assuring focality of stimulation (Deng et al. 2013).

The results of the ANOVA showed a significant interaction of TARGET $*$ ISI $[F(8,120)=2.1492, p=0.036]$. Figure 4 illustrates the interaction. This was further explored by three different univariate ANOVAs, one for each TARGET level, with ISI as sole within-subject factor. The results showed that only the ANOVA with the data from $\mathrm{P} 2$ was significant $[F(4,60)=4.49, p=0.003]$. The other $p$ s were all $>0.47$. Post hoc comparisons showed that the data at ISI $=6 \mathrm{~ms}$ were significantly different from all the other ISIs, while none of the other ISIs showed any reciprocal difference. The final analysis was to test whether any data from single ISIs were significantly different from a distribution with mean value $=0$, thereby indicating a significant effect of condTMS over testTMS. This comparison was carried out with $t$ tests for single sample. The significance threshold was set to 0.003 in order to correct for the 15 multiple comparisons. The results showed that exclusively in P2, at ISI of $6 \mathrm{~ms}$, the data were significantly different from $0[t(15)=-6.4 ; p=0.00001]$. All other $p \mathrm{~s}$

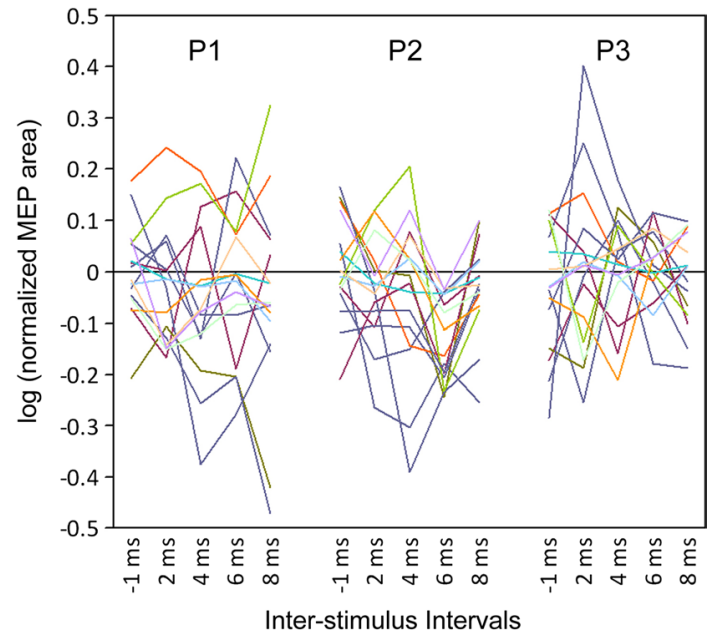

Fig. 5 Individual values of the logarithm of the normalized MEP areas. Note the robust inhibitory effect with conditioning TMS applied to P2 at an ISI of $6 \mathrm{~ms}$

Table 1 Talairach coordinates of P2 in each participant

\begin{tabular}{lrrl}
\hline Participant & \multicolumn{1}{l}{$y$} & $z$ \\
\hline$\# 1$ & -22 & -1 & 64 \\
$\# 2$ & -25 & 0 & 62 \\
$\# 3$ & -25 & -3 & 66 \\
$\# 4$ & -20 & 0 & 63 \\
$\# 5$ & -25 & -3 & 61 \\
$\# 6$ & -22 & -1 & 63 \\
$\# 7$ & -21 & -4 & 65 \\
$\# 8$ & -33 & 6 & 51 \\
$\# 9$ & -21 & 6 & 61 \\
$\# 10$ & -24 & 3 & 60 \\
$\# 11$ & -25 & 6 & 60 \\
$\# 12$ & -17 & 3 & 63 \\
$\# 13$ & -23 & 0 & 62 \\
$\# 14$ & -24 & -2 & 67 \\
$\# 15$ & -21 & 1 & 66 \\
$\# 16$ & -23 & 0 & 63 \\
Average & -23 & 1 & 62 \\
\hline
\end{tabular}

were $>0.15$. Figure 5 shows the individual values of the normalized MEP areas.

Finally, given the spatial specificity of the results, which were significant only in the $\mathrm{P} 2$ point, we described its position in a second modality, different from the anatomical one used to localize it in the first place. We transformed the brain MRIs of all participants into the Talairach space by means of the BrainVoyager software. We collected the P2 Talairach coordinates form all participants. The resulting coordinates are listed in Table 1. 


\section{Control analyses}

The baseline EMG (namely, the rectified EMG signal in the $200 \mathrm{~ms}$ prior to condTMS) was also analyzable according to the TARGET * ISI design. None of the interactions are significant for the single-pulse and baseline EMG data ( $\mathrm{min}$ $p=0.16$ ). The conventional analysis performed post hoc confirmed the main finding. Only the data from TARGET $\mathrm{P} 2$ and at ISI of $6 \mathrm{~ms}$ showed a distribution significantly different from $1(p=0.04)$. All other $p$ 's were $>0.16$.

\section{Discussion}

\section{Temporal specificity}

The present results indicate that condTMS over the PMCd exerts a powerful inhibitory effect on the corticofacial system. This effect is inhibitory and shows consistent spatial and temporal resolution. It was present exclusively for stimulation at ISI $=6 \mathrm{~ms}$ and limitedly to stimulation of P2. The temporal specificity is consistent with corticocortical connections. In fact, other studies exploring corticocortical connections between areas the distances between which are similar to that between the PMCd and the mouthM1 have found interactions at ISIs around $6 \mathrm{~ms}$. (Baumer et al. 2009; Cattaneo and Barchiesi 2011; Davare et al. 2008, 2009, 2010). It should be noted that the physical distance between the dorsal premotor cortex and the mouthM1 is of around $6 \mathrm{~cm}$, entirely compatible with a direct corticocortical connection.

However, the present data cannot exclude the possibility that the interaction between the PMC and the mouthM1 occurs at the brainstem level rather than in a corticocortical pathway, particularly given that the PMCd is known to send direct descending axons to the facial nucleus. The hypothesis that corticocortical interactions occur is nevertheless more likely for two reasons. First because the effect at 6-ms ISI would imply that corticobulbar axons from the PMCd are much slower than those from the mouthM1. The MEP onset latency in the perioral region is $\sim 10 \mathrm{~ms}$ (Cattaneo and Pavesi 2013) and the supposed latency of the corticospinal volley form the PMCd would therefore be $\sim 16 \mathrm{~ms}$. The second argument against a direct corticobulbar effect of the PMCd is the specifically inhibitory effect of the stimulation: Pyramidal neurons are supposed to be excitatory neurons.

\section{Spatial specificity}

The spatial specificity of the effect is intriguing. Where exactly is P2 on conventional brain maps? The PMCd region has been studied cytoarchitectonically in a series of human specimens in only one work (Geyer 2004). It was shown that the border between Brodmann area 6 and the prefrontal cortex (PFC) is characterized by the decreasing of the large and elongated pyramidal cells in lower layer III that are typical of area 6 . An even more important feature of the border between these two areas is the emerging granular layer (layer IV) and therefore the presence of the dysgranular cortex. Geyer (2004) showed that in the caudal part of the PFC, layer IV is discreet and not easy to detect, since the feature of the granular cortex emerges gradually rather than abruptly. The two areas somehow grade into each other; nevertheless, their border can be delineated in an observer-independent way by means of the histological processing of postmortem brains, a cytoarchitectonic analysis, as the one carried out by Geyer. Taking into account the results of this analysis, shown in Fig. 2 of Chapter 3 in his book, the assumptions made about the extension of area 6 necessary for the present study were supported. Accordingly, all three points $\mathrm{P} 1-3$ are within the PMCd, with P3 at its rostral border with PFC. Finally, it is worth noting that the spatially and temporally specific inhibitory effect described here are extremely robust, as can be observed in Fig. 5. All 16 participants were consistent in showing inhibition of the mouthM1 output.

\section{Conclusion}

In the present study, we confirm our initial experimental hypothesis. By moving the target of testTMS from the handM1 to the mouthM1, it is possible and easily feasible to assess ipsilateral PMCd-M1 circuitry by means of the dual-coil technique. This allowed us to define a specific region in the PMCd that gives origin to premotor-motor connections. The technique described offers novel possibilities for using neurostimulation as a tool to assess the physiological properties of the PMCd.

\section{Compliance with ethical standards}

Conflict of interest None.

\section{References}

Amiez C, Kostopoulos P, Champod AS, Petrides M (2006) Local morphology predicts functional organization of the dorsal premotor region in the human brain. J Neurosci 26:2724-2731

Arai N, Lu MK, Ugawa Y, Ziemann U (2012) Effective connectivity between human supplementary motor area and primary motor cortex: a paired-coil TMS study. Exp Brain Res 220:79-87

Baumer T, Schippling S, Kroeger J, Zittel S, Koch G, Thomalla G, Rothwell JC, Siebner HR, Orth M, Munchau A (2009) Inhibitory and facilitatory connectivity from ventral premotor to primary 
motor cortex in healthy humans at rest-a bifocal TMS study. Clin Neurophysiol 120:1724-1731

Brodmann K (1909) Vergleichende Lokalisationslehre der Grosshirnrinde in ihren Prinzipien dargestellt auf Grund des Zellenbaues. Johann Ambrosius Barth Verlag, Leipzig

Cattaneo L, Barchiesi G (2011) Transcranial magnetic mapping of the short-latency modulations of corticospinal activity from the ipsilateral hemisphere during rest. Front Neural Circuits 5:14

Cattaneo L, Pavesi G (2013) The facial motor system. Neurosci Biobehav Rev 38:135-159

Civardi C, Cantello R, Asselman P, Rothwell JC (2001) Transcranial magnetic stimulation can be used to test connections to primary motor areas from frontal and medial cortex in humans. Neuroimage 14:1444-1453

Davare M, Lemon R, Olivier E (2008) Selective modulation of interactions between ventral premotor cortex and primary motor cortex during precision grasping in humans. J Physiol 586:2735-2742

Davare M, Montague K, Olivier E, Rothwell JC, Lemon RN (2009) Ventral premotor to primary motor cortical interactions during object-driven grasp in humans. Cortex 45:1050-1057

Davare M, Rothwell JC, Lemon RN (2010) Causal connectivity between the human anterior intraparietal area and premotor cortex during grasp. Curr Biol 20:176-181

Deng ZD, Lisanby SH, Peterchev AV (2013) Electric field depthfocality tradeoff in transcranial magnetic stimulation: simulation comparison of 50 coil designs. Brain Stimul 6:1-13

Dum RP, Strick PL (1991) The origin of corticospinal projections from the premotor areas in the frontal lobe. J Neurosci 11:667-689

Dum RP, Strick PL (2005) Frontal lobe inputs to the digit representations of the motor areas on the lateral surface of the hemisphere. J Neurosci 25:1375-1386

Fink GR, Frackowiak RSJ, Pietrzyk U, Passingham RE (1997) Multiple nonprimary motor areas in the human cortex. J Neurophysiol 77:2164-2174

Fulton J (1935) A note on the definition of the "motor" and "premotor" areas. Brain 58:311-316

Germann J, Robbins S, Halsband U, Petrides M (2005) Precentral sulcal complex of the human brain: morphology and statistical probability maps. J Comp Neurol 493:334-356

Geyer S (2004) The microstructural border between the motor and the cognitive domain in the human cerebral cortex. Springer, Berlin

Groppa S, Werner-Petroll N, Munchau A, Deuschl G, Ruschworth MFS, Siebner HR (2012) A novel dual-site transcranial magnetic stimulation paradigm to probe fast facilitatory inputs from ipsilateral dorsal premotor cortex to primary motor cortex. Neuroimage 62:500-509

Hatanaka N, Nambu A, Yamashita A, Takada M, Tokuno H (2001) Somatotopic arrangement and corticocortical inputs of the hindlimb region of the primary motor cortex in the macaque monkey. Neurosci Res 40:9-22

He SQ, Dum RP, Strick PL (1993) Topographic organization of corticospinal projections from the frontal lobe: motor areas on the lateral surface of the hemisphere. J Neurosci 13:952-980

He SQ, Dum RP, Strick PL (1995) Topographic organization of corticospinal projections from the frontal lobe: motor areas on the medial surface of the hemisphere. J Neurosci 15:3284-3306

Johansen-Berg H, Rushworth MF, Bogdanovic MD, Kischka U, Wimalaratna S, Matthews PM (2002) The role of ipsilateral premotor cortex in hand movement after stroke. Proc Natl Acad Sci USA 99:14518-14523

Koch G, Franca M, Del Olmo MF, Cheeran B, Milton R, Alvarez Sauco M, Rothwell JC (2006) Time course of functional connectivity between dorsal premotor and contralateral motor cortex during movement selection. J Neurosci 26:7452-7459
Kraskov A, Prabhu G, Quallo MM, Lemon RN, Brochier T (2011) Ventral premotor-motor cortex interactions in the macaque monkey during grasp: response of single neurons to intracortical microstimulation. J Neurosci 31:8812-8821

Mars RB, Klein MC, Neubert FX, Olivier E, Buch ER, Boorman ED, Rushworth MF (2009) Short-latency influence of medial frontal cortex on primary motor cortex during action selection under conflict. J Neurosci 29:6926-6931

Maule F, Barchiesi G, Brochier T, Cattaneo L (2015) Haptic working memory for grasping: the role of the parietal operculum. Cereb Cortex 25:528-537

Mochizuki H, Terao Y, Okabe S, Furubayashi T, Arai N, Iwata NK, Hanajima R, Kamakura K, Motoyoshi K, Ugawa Y (2004) Effects of motor cortical stimulation on the excitability of contralateral motor and sensory cortices. Exp Brain Res 158:519-526

Morecraft RJ, Louie JL, Herrick JL, Stilwell-Morecraft KS (2001) Cortical innervation of the facial nucleus in the non-human primate: a new interpretation of the effects of stroke and related subtotal brain trauma on the muscles of facial expression. Brain 124:176-208

Muakkassa KF, Strick PL (1979) Frontal lobe inputs to primate motor cortex: evidence for four somatotopically organized 'premotor' areas. Brain Res 177:176-182

O'Shea J, Johansen-Berg H, Trief D, Gobel S, Rushworth MF (2007a) Functionally specific reorganization in human premotor cortex. Neuron 54:479-490

O'Shea J, Sebastian C, Boorman ED, Johansen-Berg H, Rushworth MF (2007b) Functional specificity of human premotor-motor cortical interactions during action selection. Eur $\mathrm{J}$ Neurosci 26:2085-2095

Rizzolatti G, Luppino G, Matelli M (1998) The organization of the cortical motor system: new concepts. Electroencephalogr Clin Neurophysiol 106:283-296

Rossi S, Hallett M, Rossini PM, Pascual-Leone A (2009) Safety, ethical considerations, and application guidelines for the use of transcranial magnetic stimulation in clinical practice and research. Clin Neurophysiol 120:2008-2039

Rothwell JC (2011) Using transcranial magnetic stimulation methods to probe connectivity between motor areas of the brain. Hum Mov Sci 30:906-915

Sato M, Buccino G, Gentilucci M, Cattaneo L (2010) On the tip of the tongue: modulation of the primary motor cortex during audiovisual speech perception. Speech Commun 5:2533-2541

Schluter ND, Rushworth MF, Passingham RE, Mills KR (1998) Temporary interference in human lateral premotor cortex suggests dominance for the selection of movements. A study using transcranial magnetic stimulation. Brain 121(Pt 5):785-799

Tokuno H, Tanji J (1993) Input organization of distal and proximal forelimb areas in the monkey primary motor cortex: a retrograde double labeling study. J Comp Neurol 333:199-209

Tukey JW (1977) Exploratory data analysis. Addison-Wesley, Reading, MA

Vogt C, Vogt O (1919) Allgemeine Ergebnisse unserer Hirnforschung. Journal für Psychologie und Neurologie 25:277-462

Watkins K, Paus T (2004) Modulation of motor excitability during speech perception: the role of Broca's area. J Cogn Neurosci 16:978-987

Watkins KE, Strafella AP, Paus T (2003) Seeing and hearing speech excites the motor system involved in speech production. Neuropsychologia 41:989-994

World Medical Association General Assembly (2008) Declaration of Helsinki. Ethical principles for medical research involving human subjects. World Med J 54:122-125 